\title{
Mass-Spring-Damping Theory Based Equivalent Mechanical Model for Cylindrical Lithium-ion Batteries under Mechanical Abuse
}

Wenwei Wang ${ }^{1,2}$, Yiding $\mathrm{Li}^{1,2^{*}} \mathbb{0}$, Cheng Lin ${ }^{1,2}$ and Sheng Yang ${ }^{1,2}$

\begin{abstract}
An equivalent mechanical model with the equivalent physical meaning of mass-spring-damping is proposed for cylindrical lithium-ion batteries through experiments and theory. The equivalent mechanical model of a cylindrical lithium-ion battery consists of a spring-damping parallel unit. Therefore, a spring-damping parallel unit connecting a damping unit in series is selected to construct the constitutive characteristics of the battery under mechanical abuse. Comparison results show that the equivalent mechanical model can more effectively describe the mechanical properties of the batteries than most cubic fitting models, of which the average relative error of the equivalent mechanical model under different states-of-charge is less than 6.75\%. Combined with the proposed equivalent mechanical model, the failure process of the batteries was simulated and analyzed using LS-Dyna and HyperWorks. Under rigid rod tests, failure occurred at the core and bottom of the batteries; under hemispherical punch tests, failure occurred at the core and top, consistent with the experimental results. The average prediction error for the failure displacement under different abuse conditions is less than $4 \%$ in the simulations. The equivalent mechanical model requires only a few parameters and can be recognized easily. In the future, the model can be used in safety warning devices based on mechanical penetration.
\end{abstract}

Keywords: Cylindrical lithium-ion battery, Mass-spring-damping theory, Equivalent mechanical model, Mechanical abuse condition, Failure analysis

\section{Introduction}

With the development of electric vehicles, the safety of lithium-ion batteries has become a societal concern [1, 2]. In the last two years, several safety accidents involving lithium-ion battery systems that resulted in severe casualties in electric vehicles have occurred. Based on safety reasons, China has launched a new car assessment program (C-NCAP) in 2018, in which requirements for the crash performance of pure electric vehicles have been added. Therefore, the mechanical properties of lithiumion batteries under mechanical abuse conditions must

\footnotetext{
*Correspondence: liyid00@126.com

${ }^{1}$ National Engineering Laboratory for Electric Vehicles, Beijing Institute

of Technology, Beijing 100081, China

Full list of author information is available at the end of the article
}

be understood comprehensively to as a foundation for future battery safety studies.

A lithium-ion power battery comprises a positive electrode made of a positive active material and a positive current collector; and a negative electrode made of a negative active material, as well as a negative current collector and separator. Lithium-ion battery failure often occurs when a separator is damaged and the anode is in contact with the cathode, which causes a short circuit. Hence, most studies focus on the mechanical properties of the separator [3-5].

Some researchers have studied the mechanical properties of cylindrical lithium-ion cells subjected to mechanical abuse conditions and corresponding stress-strain constitutive models have been proposed. In the mechanical modeling of lithium-ion batteries, the force-displacement response is generally summarized as a cubic fitting 
equation. Wierzbicki and Avdeev et al. [6, 7] established a cubic fitting equation for cylindrical lithium-ion batteries under various load conditions and proposed a set of methods to calculate the average stress-strain. Based on the cubic fitting model, in-depth studies regarding the constitutive mechanical properties of the battery have been conducted; for example, the clay-like theory by Wang et al. [8] and the principle of virtual work by Sahraei et al. [9]. The cubic fitting model can predict the force-displacement response of lithium-ion batteries. Zhang et al. [10] proposed a constitute behavior at the electrode level to describe the mechanical failure of lithium-ion batteries; the model provided insights for improving the structure strength of lithium-ion batteries. Chung et al. [11] conducted various abuse tests to discuss the critical fracture, and the Mohr-Coulomb criterion was used to predict the failure of the batteries. Xu et al. [12] obtained the plastic stress-strain of the lithium-ion batteries using the Johnson-Cook material model.

Although the cubic fitting equation has a simple expression form and is convenient for mechanical modeling, it does not offer a high precision, which will result in large errors.

The stress field and structural damage of batteries under mechanical abuse through finite element simulation analysis can be obtained. Combined with simulation analysis, constitutive mechanical models can well predict the deformation behavior of cells under mechanical loads. The crushable-foam material model is a useful homogenized modeling method in which the battery is considered in entirety and evaluated from a macroscopic perspective [6-9, 13-15]. Homogenized modeling is simple, but it cannot reflect the actual mechanical changes inside the cells. Therefore, another method has been established to model the cathode, anode, and separator of the battery, separately [16-18]. Additionally, the representative volume element (RVE) method can be used to model lithium-ion batteries. Zhang et al. [10, 19] and other researchers have used the RVE model to calculate the mechanical properties of batteries, and their results were agreed well with experimental results. Liu et al. [20] developed a multiphysics computational model based on the RVE method, and a full spectrum safety mechanism map was proposed.

Homogenized models are the most computationally efficient, and the detailed models include the most information but are computationally intensive; the performance of the RVE method is in between those of the abovementioned methods [21].

The State-of-Charge (SOC) can change the mechanical properties and the volume of active particles owing to the expansion and contraction of the anode and cathode; therefore, the mechanical properties of the cells will change. Hence, we may conclude that the SOC can significantly change the properties of hard-shell batteries [22-25, 30].

By studying the magnitude-phase-angle characteristics of batteries, an equivalent mechanical model with equivalent physical significance is proposed based on the mass-spring-damping theory. The configuration of a spring-damping parallel unit connecting a damping unit in series was selected and modified. Combined with the crushable-foam material model and HyperWorks/LSDyna, we simulated and verified the proposed model. The simulation results can explain the failure behavior of the battery well, i.e., under rigid rod tests, the failure of the batteries occurs at the core and bottom, while under hemispherical punch tests, failure occurs at the core and top. The average prediction error of the modified equivalent mechanical model to the failure displacement under different load conditions is less than $4 \%$. In the future, the equivalent mechanical model can be conveniently used in battery safety warning equipment under the condition of mechanical abuse.

\section{Materials and Methods}

\subsection{Experimental Equipment and Process}

The experimental settings are shown in Figure 1. Sony VTC4 18650 cylindrical lithium-ion batteries with nominal capacity $2100 \mathrm{mAh}$ were used in the experiments, as shown in Figure 1c. Constant current-constant voltage was applied to discharge and charge the batteries to $\mathrm{SOC}=0,0.2,0.4,0.5,0.6$, and 0.8. An INSTRON universal tensile testing machine with the maximum load of

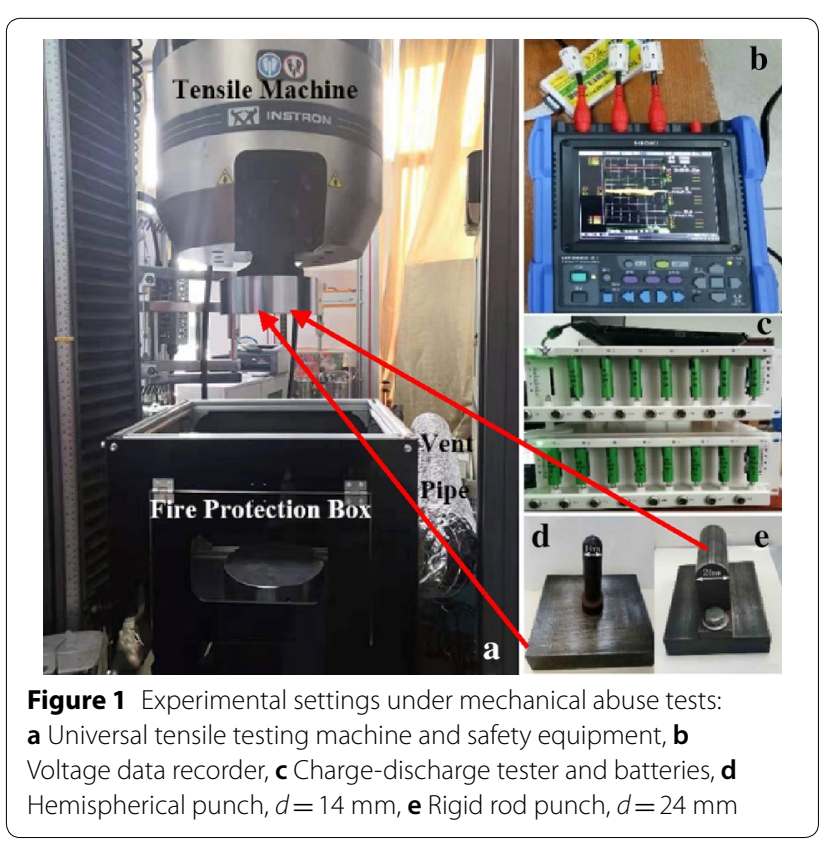


$250 \mathrm{kN}$ was used to compress the batteries, as shown in Figure 1a. A fire protection box was used to protect the test equipment and limit smoke and fire from battery explosions into a confined environment, and a vent pipe was used to process the smoke and channel it outside. A four-channel HOKI MR8880 data recorder was used, as shown in Figure 1b, as well as a FLUKE Ti400 infrared thermal camera.

The mechanical abuse tests performed in this study included flat plate, rigid rod, and hemispherical punch tests. The diameters of the hemispherical and rigid rod punches were 14 and $24 \mathrm{~mm}$, respectively, as shown in Figure $1 \mathrm{~d}$ and e. A lower penetration speed can produce more adverse results [26]; therefore, the mechanical abuse experiments in this study were performed under the quasi-static conditions at the loading speed of $0.5 \mathrm{~mm} / \mathrm{min}$. During the mechanical abuse experiments, the force-displacement, voltage, and temperature were recorded simultaneously.

\subsection{Mathematical Model Description: Mass-Spring-Damping System}

In a sense, the essence of mechanical system is the massspring-damping system. Under mechanical abuse conditions, the mechanical response of the cells can be treated as a mass-spring-damping system.

In the time domain, the expression of force to displacement for the mass-spring-damping system is complicated. However, in the frequency domain, when the equivalent spring stiffness of mass, spring, and damping units are known, the relationship between force and displacement can be derived easily.

The equivalent spring stiffness of mass quality $M$, spring elastic stiffness $k$, damping coefficient $D$, and corresponding structures are shown in Table 1. $f(t)$ and $F(s)$ are forces in the time and frequency domains, respectively. $x(t)$ and $X(s)$ are displacements in the time and frequency domains, respectively. $s$ is the symbol representing the inverse of time, i.e., $s=\omega j$, where $\omega$ is the angular frequency, and $j$ is the imaginary part.

The mass-spring-damping system can be composed of a single or two or more components connected in series or parallel to form an integrated mass-spring-damping system:

$$
\begin{aligned}
& \frac{f(j \omega)}{x(j \omega)}=\sum_{n=1}^{z} \frac{f(t)}{x(t)} e^{-j \omega t}, \\
& H(j \omega)_{n}=a_{n}+b_{n} j, \\
& \left|A_{m}\right|_{n}=\sqrt{a_{n}^{2}+b_{n}^{2}}, \\
& \omega_{n}=\arctan \frac{b_{n}}{a_{n}} .
\end{aligned}
$$

A Fourier transform on the experimental data of the flat plate, rigid rod, and hemispherical punch tests was performed. As shown in Eqs. (1)-(4), $n$ is the series of the discrete time signal, $H(j \omega)_{n}$ is the transfer function, $a_{n}$ and $b_{n}$ are the coefficients, $\left|A_{\omega}\right|_{n}$ is the magnitude, and $\omega_{n}$ is the phase angle.

As shown on the right side of Figure 2, from the qualitative analysis of the experiment, the mechanical characteristics of the batteries under different mechanical abuse conditions in the limited frequency range contained the

\begin{tabular}{|c|c|c|c|c|}
\hline Component & Structure model & Time-domain equation & $\begin{array}{l}\text { Frequency-domain equation } \\
\text { (L) }\end{array}$ & $\begin{array}{l}\text { Equivalent } \\
\text { spring } \\
\text { stiffness }\end{array}$ \\
\hline Mass & & $f(t)=M \ddot{x}(t)$ & $F(s)=M s^{2} X(s)$ & $M s^{2}$ \\
\hline Spring & & $f(t)=k x(t)$ & $F(s)=k X(s)$ & k \\
\hline Damping & & $f(t)=D \dot{x}(t)$ & $F(s)=D s X(s)$ & Ds \\
\hline
\end{tabular}
spring-damping parallel unit, as shown on the left of Figure 1 . This suggests that the mechanical properties of the

Table 1 Equivalent spring stiffness of the components 


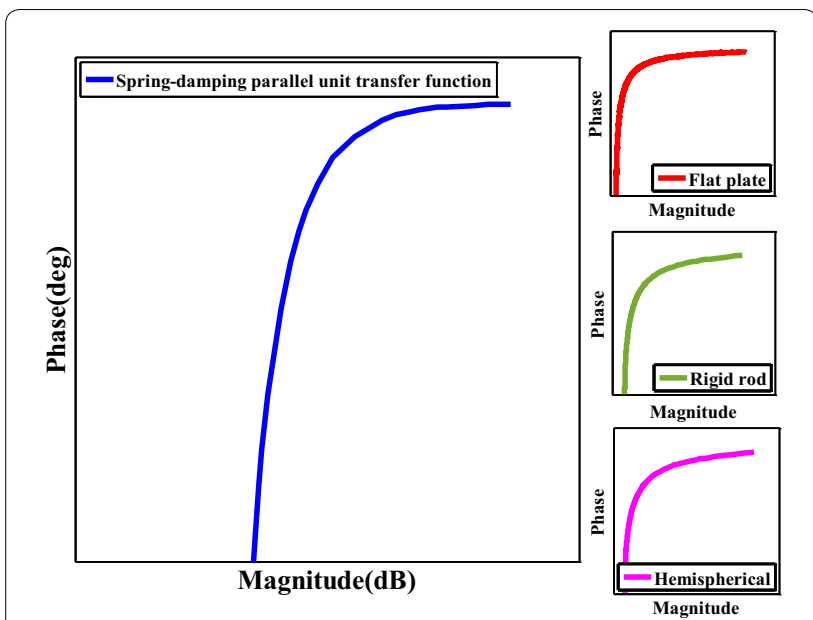

Figure 2 Magnitude-Phase-angle characteristics of the battery under mechanical abuse conditions in frequency domain

batteries can be simplified by an equivalent mechanical model that contains a spring-damping parallel unit. In other words, the cylindrical lithium-ion batteries exhibit the equivalent physical effect of the spring-damping parallel unit under mechanical abuse conditions.

Based on the findings above, three different forms of equivalent mechanical models were analyzed and validated.

In all of the equivalent mechanical models, the action end of the force is free, and the component is unconstrained.

\subsubsection{Configuration 1: Spring-Damping Parallel Structure}

The Configuration 1 structure shown in Figure 3 is the basic spring-damping parallel unit. In the frequency domain, the response of the spring-damping parallel system, which is the displacement to the force, is given by Eq. (5):

$$
f(s)=(k+D s) X(s)
$$

Subsequently, Eq. (5) can be converted to a transfer function that is related to the force and displacement:

$$
H(s)=\frac{f(s)}{X(s)}=k+D s .
$$

To obtain the equivalent equation in the time domain, an inverse Laplace transform is performed to Eq. (7):

$$
\frac{x(t)}{f(t)}=L^{-1}[H(s)]=L^{-1}\left[\frac{X(s)}{f(s)}\right]=\frac{e^{-\frac{k}{D} t}}{D},
$$
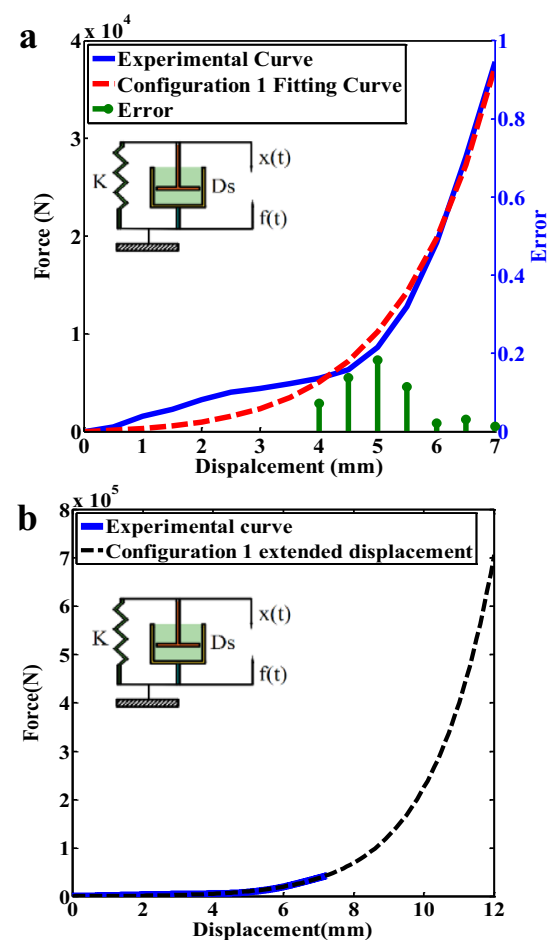

Figure 3 Configuration 1: equivalent mechanical model and verification results: a results of experiments and Eq. (9), b verification of the extended displacement of Eq. (9)

$$
f(t)=\frac{D}{e^{-\frac{k}{D} t}} x(t) .
$$

In Eq. (7), $H(s)$ is the force-displacement transfer function in the frequency domain, $L^{-1}$ the symbol of the inverse Laplace transform, and $v$ the simulation loading speed.

In the experiment, we did not consider the effect of time; therefore, the time $t$ was replaced by the compression displacement $x$ and compression speed $v$, $x=v t \rightarrow t=\frac{x}{v}$. The force-displacement equation of the spring-damping parallel system is shown in Eq. (9):

$$
f(x)=\frac{D}{e^{-\frac{k x}{D v}}} x .
$$

To verify the effectiveness of Eq. (9), the result for flat plate compression under $\mathrm{SOC}=0$ was selected, and the equation for the extended displacement was tested. The corresponding parameters are shown in Table 2, where $v$ was set to $100 \mathrm{~mm} / \mathrm{s}$, which is the simulation speed rather than the actual loading speed of $0.5 \mathrm{~mm} /$ min that would cause the equation to be distorted in advance. The validity and applicability results are shown in Figure 3a and b, respectively. 
Table 2 Equation parameter of Eq. (9)

\begin{tabular}{llll}
\hline Fitting parameter & $\boldsymbol{D}$ & $\boldsymbol{k}$ & $\boldsymbol{v}(\mathbf{m m} / \mathbf{s})$ \\
\hline Value & 184.5 & 8866 & 100 \\
\hline
\end{tabular}

As shown in Figure 3a, the curve obtained using Eq. (9) at the region after $4 \mathrm{~mm}$ can reflect the experimental data well; the red curve is coincident with the experimental data, the average relative error between the Configuration 1 model and the experiment is less than $8.2 \%$, and correlation coefficient $\mathrm{R}^{2}$ is 0.9938 . However, in the first half of the curve, the equation cannot reflect the experiment because the compression primarily compresses the space in the battery, e.g., the electrolyte, gap between parts, etc. At this stage, the mechanical properties of the battery are similar to those of the spring unit; therefore, the force exhibits an approximately linear growth. However, Figure $3 \mathrm{~b}$ shows that when the displacement is larger than the experimental area, in which the displacement increases by only $3 \mathrm{~mm}$, the force increases significantly. The force value increased by one order of magnitude. This type of growth can be considered as inconsistent with the actual load condition. Therefore, the basic spring-damping parallel unit cannot fully describe the properties of cylindrical lithium-ion batteries.

\subsubsection{Configuration 2: Mass-Spring-Damping Structure}

The structure of Configuration 2 is that a mass unit is connected in series before the spring-damping parallel part, as shown in Figure 4. In the frequency domain, the displacement to force mass-spring-damping system is given by Eq. (10):

$$
\begin{aligned}
& f(s)=\frac{1}{\frac{1}{m s^{2}}+\frac{1}{k+D s}} X(s)=\frac{m D s^{3}+m k s^{2}}{m s^{2}+D s+k} X(s), \\
& \frac{x(t)}{f(t)}=L^{-1}[H(s)]=L^{-1}\left[\frac{1}{m s^{2}}+\frac{1}{k+D s}\right]=\frac{m e^{-\frac{k}{D} t}+D t}{m D}, \\
& f(x)=\frac{m D}{m e^{-\frac{k}{D} \frac{x}{v}}+D \frac{x}{v}} x .
\end{aligned}
$$

Similar to Method 1, the comparison and applicability of Configuration 2 and Eq. (12) are shown in Figure 4a and $b$, of which the parameters are shown in Table 3.

By comparison, the results show that although Configuration 2 can reflect the basic mechanical properties of the battery, the average relative error between the experimental results and Configuration 2 model after $4 \mathrm{~mm}$ is less than $7 \%$ and the correlation coefficient $\mathrm{R}^{2}$ is 0.9947 . However,
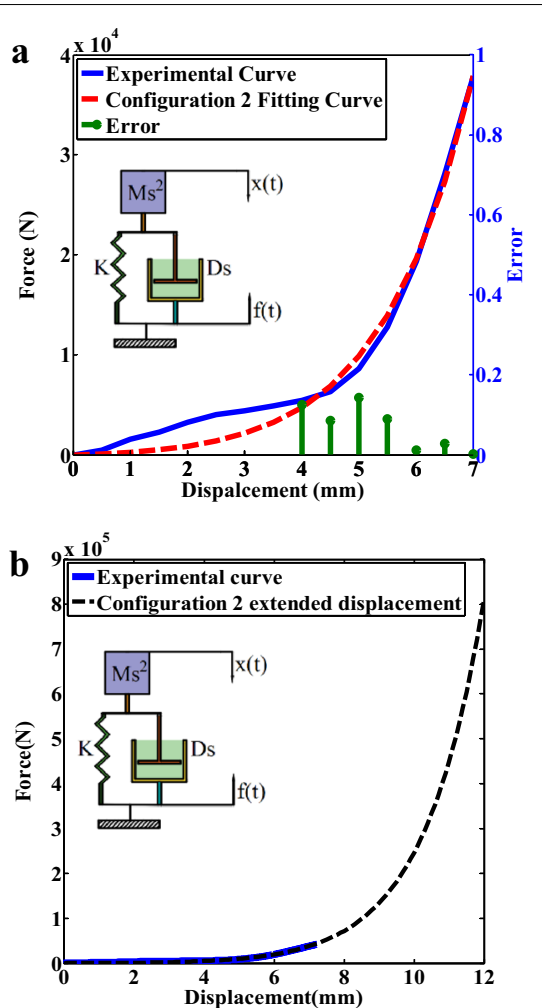

Figure 4 Configuration 2 equivalent mechanical model and verification results: a Results of experimental data and Eq. (12), b Verification of extended displacement of Eq. (12)

the problem where the force increases excessively after an extended displacement still exists. Hence, we did not use this method for our application.

\subsubsection{Configuration 3: Spring-Damping Parallel Unit Connects a Damping Unit in Series}

Configuration 3 is the damping unit connected in series after the spring-damping parallel unit, as shown in Figure 5. In the frequency domain, the force to displacement relationship in frequency domain is given by Eq. (13):

$$
\begin{aligned}
& f(s)=\frac{1}{\frac{1}{D_{2} s}+\frac{1}{k+D_{1} s}} X(s)=\frac{D_{1} D_{2} s^{2}+k D_{2} s}{\left(D_{1}+D_{2}\right) s+k} X(s), \\
& \frac{x(t)}{f(t)}=L^{-1}\left[\frac{X(s)}{f(s)}\right]=\frac{D_{2} e^{-\frac{k}{D_{1}} t}+D_{1}}{D_{1} D_{2}} .
\end{aligned}
$$

Table 3 Equation parameter of Eq. (12)

\begin{tabular}{lllll}
\hline Fitting parameter & $\boldsymbol{D}$ & $\boldsymbol{k}$ & $\boldsymbol{m}$ & $\boldsymbol{v}(\mathbf{m m} / \mathbf{s})$ \\
\hline Value & 157.5 & 7958 & $3.729 \times 10^{8}$ & 100
\end{tabular}



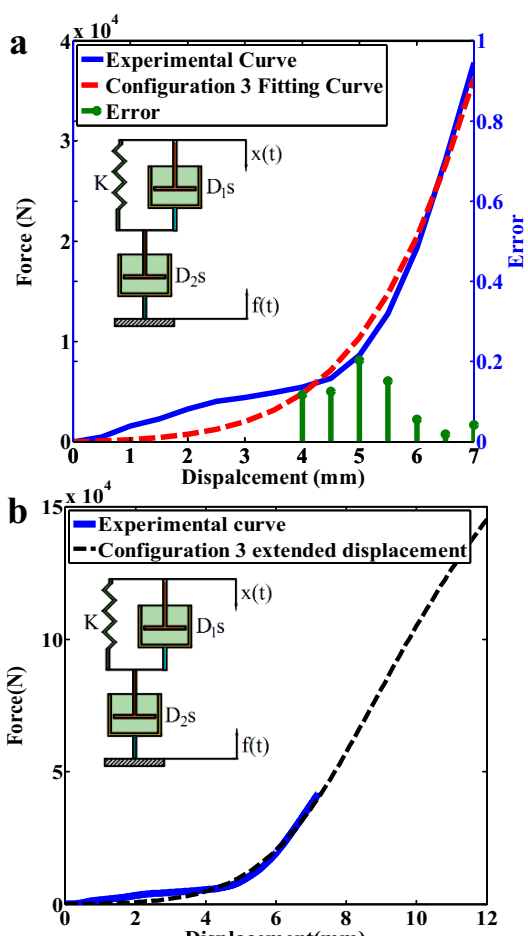

Figure 5 Configuration 3 equivalent mechanical model and verification results: a Results between experimental data and Eq. (15), b Verification of extended displacement of Eq. (15)

Table 4 Equation parameter setting of Eq. (15)

\begin{tabular}{lllll}
\hline Fitting parameters & $\boldsymbol{D}_{\mathbf{1}}$ & $\boldsymbol{D}_{\mathbf{2}}$ & $\boldsymbol{k}$ & $\boldsymbol{v}(\mathbf{m m} / \mathbf{s})$ \\
\hline Value & 108.2 & $1.29 \times 10^{4}$ & 6772 & 100 \\
\hline
\end{tabular}

After an inverse Laplace transform, one obtains Eq. (15):

$$
f(x)=\frac{D_{1} D_{2}}{D_{2} e^{-\frac{k}{D_{1}} \frac{x}{v}}+D_{1}} x .
$$

The corresponding parameters and comparison of Eq. (15) are shown in Table 4, and Figure 5a and b, respectively.

As shown, this structure exhibits good characteristics in the second half; the average relative error between the experimental results and Configuration 3 model is less than $10 \%$ and the correlation coefficient $R^{2}$ is 0.9910 . Similar to the two schemes mentioned above, this solution contains errors in the first stage of the curve. To solve this problem, the piecewise function will be used, which will be discussed in Section 2.3. The verification of suitability for the extended displacement shows that the increase in force is less than those of the first two structures mentioned previously, and that the model is more consistent with the actual situation; therefore, it has a higher application value. Hence, we will use the Configuration 3 model for our subsequent study.

The parameter analysis suggests that cylindrical lithium-ion batteries are composed of a spring-damping parallel unit and a damping unit connected in series in terms of the equivalent physics. This can simplify the mechanical model of the battery based on considering the structure and render the relevant parameters easy to identify. It would be beneficial to construct the corresponding finite element simulation.

\subsection{Optimization of the Equivalent Mechanical Model under Multi-SOC}

To verify the adaptation of the proposed Configuration 3 structure under various SOC states, several SOC states with $\mathrm{SOC}=0,0.2,0.4,0.5,0.6$, and 0.8 were selected and tested under the condition of flat plate compression and then compared with the most frequently used cubic fitting Eqs. (6)-(9). The proposed equivalent model was modified accordingly to improve the performance of the entire compression process.

To ensure the repeatability of the experiment, the tests were repeated several times to obtain similar results with the best correlation of the experimental data. The forcedisplacement equation derived from Configuration 3 was calculated and compared with the cubic fitting equation of Eq. (16):

$$
f(x)=c \times x^{3} .
$$

As mentioned previously, the result of Configuration 3 before $4 \mathrm{~mm}$ was not ideal. The deviation was primarily due to the gap between battery parts. Therefore, the equation of Configuration 3 should be modified. From the experiment, it was discovered that the growth of the force was almost linear in the first half (before $4 \mathrm{~mm}$ ) of the curve. We have discussed this in Section 2.2.1 where, in the first half of the compression process, the main reason for the linear growth is the force squeezing the gap between the battery components. Hence, the equivalent mechanical model can be modified by a piecewise function that, in the first half of the model, modified the function by a linear equation, and expressed the progress by the Configuration 3 equation in the second half of the model. Discrete force-displacement and stress-strain relationships are required in the model validation and simulations; therefore, the linear interpolation algorithm was used near the critical turning displacement $x_{l}$ to ensure the continuity of the modified equivalent mechanical equation. 
The basic form of the modified equivalent mechanical equation is shown in Eq. (17):

$$
f(x)= \begin{cases}k_{t} x, & 0 \leq x \leq x_{l}, \\ \frac{D_{1} D_{2}}{D_{2} e^{-\frac{k}{D_{1}} \frac{x}{v}}+D_{1}} x, & x>x_{l},\end{cases}
$$

where $k_{t}$ represents the slope of the linear increasing section, and $x_{l}$ is the critical turning displacement of the linear section and the Configuration 3 equation.

The experimental and equivalent model results are optimized based on Eq. (17). The parameters are shown in Table 5, and the comparison results are shown in Figure $6 \mathrm{a}-\mathrm{f}$.

In Figure $6 \mathrm{a}-\mathrm{f}$, the black needle line is the error between the cubic fitting equation and the experiment, and the red needle line is the error between the modified equivalent model and the experiment. The results show that the prediction error of the modified equivalent model is much smaller than that of the cubic fitting equation in the nonlinear part. Under the flat plate tests, the relative errors among the equivalent model, cubic fitting equation, and experiment are shown in Table 6.

The average prediction error of the equivalent model to the experiment is less than $6.75 \%$, and the correlation coefficient of the data is greater than 0.9976 .

As shown, the piecewise equivalent mechanical model of Configuration 3 can effectively predict the experimental data under various SOCs.

Under the same compression direction, the mechanical properties of the battery will not change; the simulations of the flat plate, rigid rod, and hemispherical punch tests will be discussed in the following sections. Additionally, the experimental phenomena will be explained along with the simulation results to reveal the failure characteristics of the battery.

\begin{tabular}{|c|c|c|c|c|c|}
\hline SOC & $k_{t}$ & $D_{1}$ & $D_{2}$ & $k$ & c \\
\hline 0 & 1345.7 & 108.2 & $1.290 \times 10^{4}$ & 6772 & 106.2 \\
\hline 0.2 & 1391.4 & 213.8 & $7.322 \times 10^{7}$ & 10020 & 105.5 \\
\hline 0.4 & 1461.9 & 269.9 & $8.732 \times 10^{7}$ & 12000 & 114.7 \\
\hline 0.5 & 1358.0 & 31.72 & $9.205 \times 10^{3}$ & 2967 & 125.8 \\
\hline 0.6 & 1451.1 & 67.52 & $1.018 \times 10^{4}$ & 5724 & 146.0 \\
\hline 0.8 & 1586.0 & 32.82 & $1.032 \times 10^{3}$ & 3288 & 153.9 \\
\hline
\end{tabular}

\section{Results and Discussion}

\subsection{Simulation and Verification}

According to Refs. [6, 8], the stress-strain constitutive properties of cylindrical lithium-ion batteries can be calculated by the average stress-strain method under flat plate compression. An example of a flat plate compression, the corresponding dimensions, and its meaning are shown in Figure 7a. During the calculation, we applied the following assumptions [8]:

- During the compression process, the cylindrical lithium-ion batteries and its contact surfaces remained flat.

- The total length of the cells remained unchanged during compression.

Furthermore, Ref. [6] summarized that the average stress-strain of the batteries can be expressed by Eqs. (18)-(20):

$$
\begin{aligned}
& b=\frac{\pi}{4} x, \\
& \sigma_{a v}=\frac{f(x)}{2 b L}, \\
& \varepsilon_{a v}=\frac{x}{2 R},
\end{aligned}
$$

where $\sigma_{a v}$ is the average stress, $\varepsilon_{a v}$ the average strain, $b$ the width of the compression plane, and $L$ the effective compression length of the battery, where $L=58 \mathrm{~mm}$ when the positive electrode of the battery was removed. We can use part of the battery length rather than the entire length. Wierzbicki et al. concluded that the contributions from the shell casing and end-caps to resist deformation could be neglected, compared with the jellyroll $[6,9] . R$ is the radius before the battery was compressed, the measured value of which was $R=8.8 \mathrm{~mm}$.

In the simulation experiments, the thermal softening effect on the battery was not considered in the mechanical model because the compression duration was short [14, 27, 28].

The crushable-foam material model is the most frequently used model in battery modeling $[6,9,14,19]$. We adopted the typical self-similar yield surface model presented by Deshpande et al. [29] to predict the stress-strain relationship. The yield function is given in Eq. (21), where $A$ is the equivalent stress, $\alpha$ the shape of the yield surface, $\sigma_{e}$ the von Mises effective stress calculated by Eq. (23), $\sigma_{m}$ the mean stress following Eq. (24), and $Y$ the uniaxial yield strength in compression, also known as the tensile cutoff value in simulations. In the simulation, using Eqs. (21)-(24), 
a

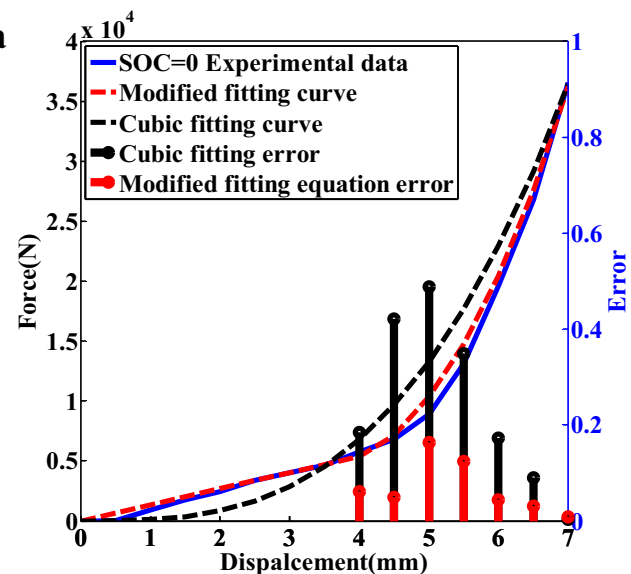

c

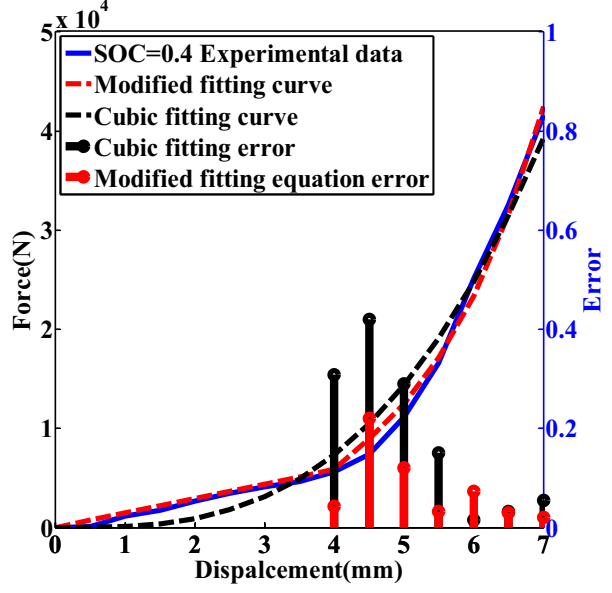

e $3.5 \times 10^{4}$

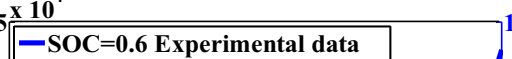

- - Modified fitting curve

3 - - Cubic fitting curve

$\rightarrow$ Cubic fitting error

2.5 Modified fitting equation error

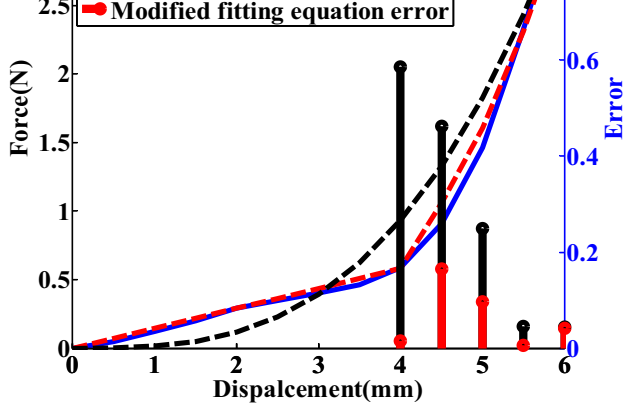

b

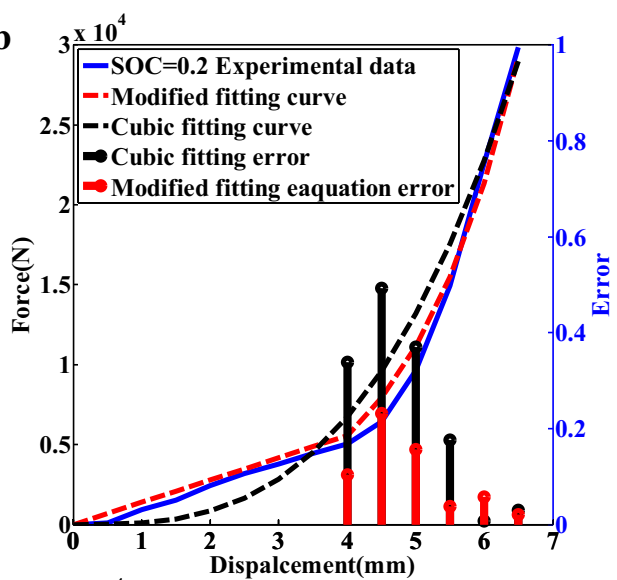

d

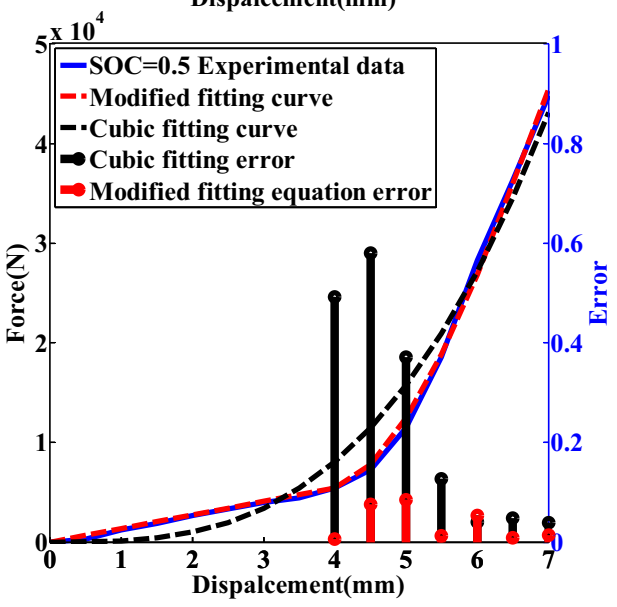

f

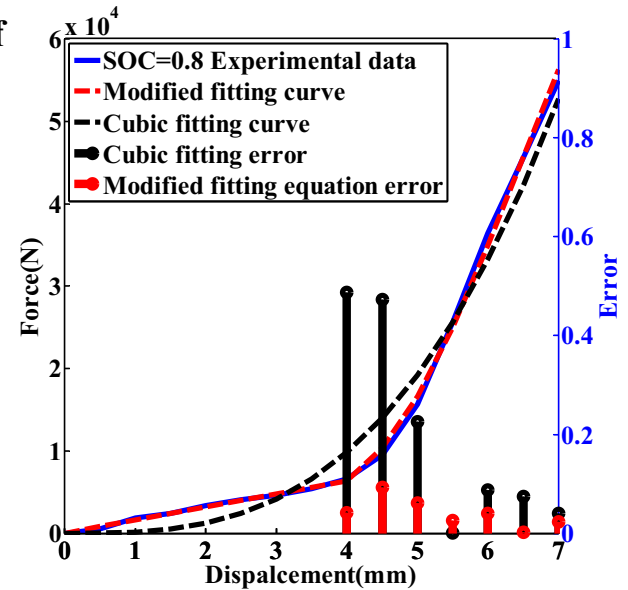

Figure 6 Comparison of equivalent mechanical model with cubic fitting equation under multi-SOC states. Comparison results of flat plate test under $\mathbf{a} \mathrm{SOC}=0, \mathbf{b} \mathrm{SOC}=0.2, \mathbf{c} \mathrm{SOC}=0.4, \mathbf{d} \mathrm{SOC}=0.5, \mathbf{e} \mathrm{SOC}=0.6$, and $\mathbf{f} \mathrm{SOC}=0.8$

Table 6 Relative error and correlation coefficient $R^{2}$ among equivalent model, cubic fitting equation and experiment

\begin{tabular}{|c|c|c|c|c|c|c|}
\hline SOC & 0 & 0.2 & 0.4 & 0.5 & 0.6 & 0.8 \\
\hline Relative error between equivalent model and experiment (\%) & 6.89 & 10.10 & 7.69 & 3.63 & 6.49 & 5.72 \\
\hline Correlation coefficient between equivalent model and experiment & 0.9985 & 0.9976 & 0.9981 & 0.9993 & 0.9977 & 0.9993 \\
\hline Relative error between cubic fitting equation and experiment (\%) & 24.43 & 23.56 & 18.15 & 24.23 & 27.74 & 27.78 \\
\hline
\end{tabular}




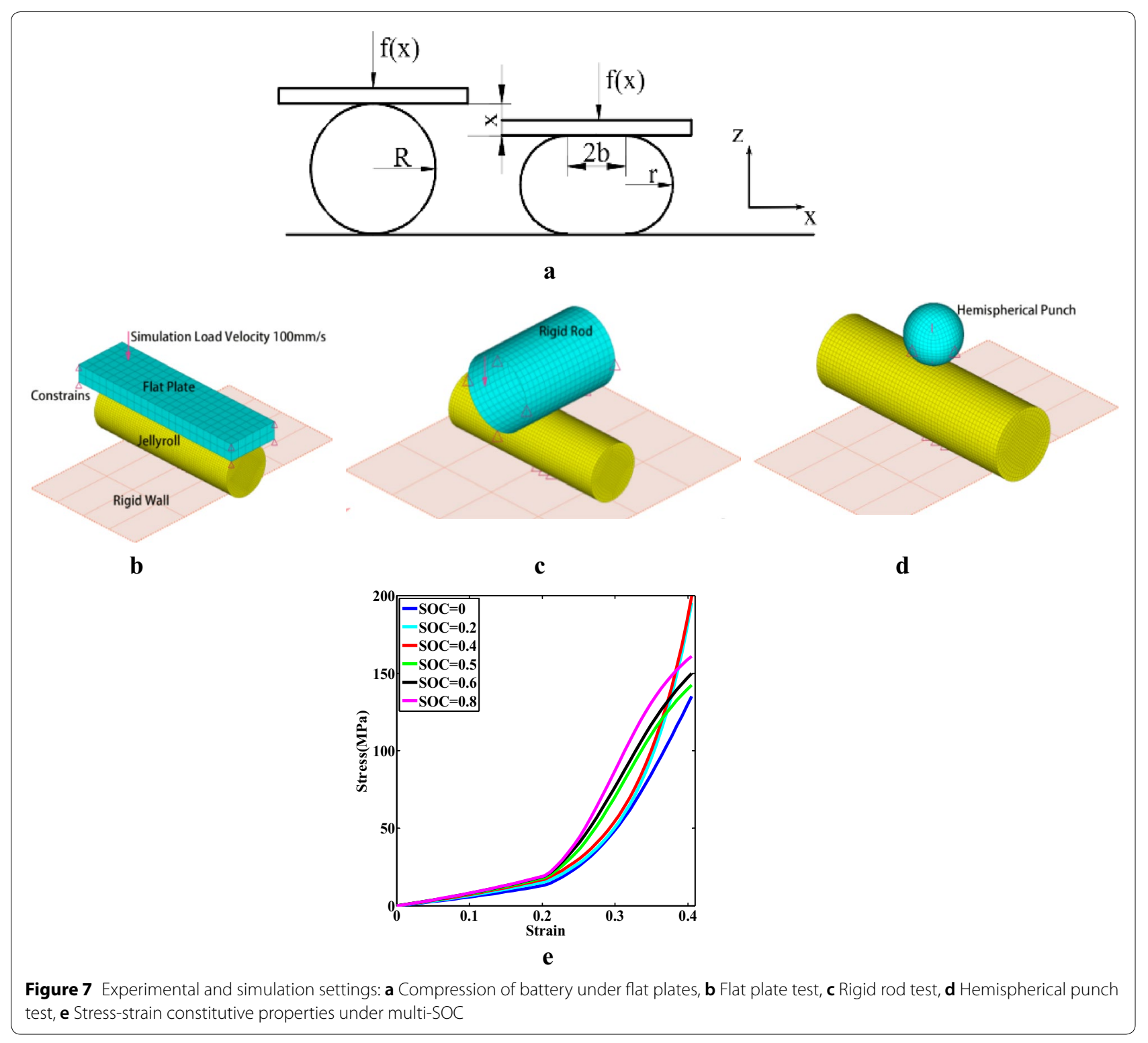

the failure elements were shown to be under mechanical abuse; therefore, we can acquire the damaged structure.

$$
\begin{aligned}
& \Lambda=\hat{A}-Y \leq 0, \\
& \hat{A}^{2}=\frac{1}{\left[1+(\alpha / 3)^{2}\right]}\left(\sigma_{e}^{2}+\alpha^{2} \sigma_{m}^{2}\right), \\
& \sigma_{e}=\sqrt{\frac{3}{2} \sigma_{i j}^{\prime} \sigma_{i j}^{\prime}}, \\
& \sigma_{m}=\frac{\sigma_{i i}}{3} .
\end{aligned}
$$

The material model requires two key inputs: stressbody strain datasets and tensile stress cutoff value (TSC). The real stress-strain value shown in Figure 7e can be used as the stress-body strain, and the TSC is $10 \mathrm{MPa}$. The simulation elements are shown in Figure $7 \mathrm{~b}-\mathrm{d}$. In LS-Dyna, the properties are solid and the material model is crushable-foam for jellyroll. The properties and material for the flat plate are solid and rigid, while those for the rigid rod and hemispherical punch are shell and rigid. The jellyroll was divided with a mesh size of $1 \mathrm{~mm}$ and 46200 elements. If the simulation velocity is $0.5 \mathrm{~mm} / \mathrm{min}$, then the compression speed will yield an unacceptable calculation time. Meanwhile, the high compression speed can be 
regarded as a quasi-static state, in which the kinetic energy of the punch in the simulation can be ignored [17]. Hence, to reduce the calculation time, $100 \mathrm{~mm} / \mathrm{s}$ was set as the compression speed. After the simulation, it was discovered that the actual kinetic energy of the punch affected the total energy insignificantly, i.e., less than $1 \%$; therefore, the high compression speed could be used to simulate the quasi-static process.

Combined with Eqs. (18)-(20), the stress-strain for cylindrical lithium-ion batteries is shown in Eq. (26):

$$
\begin{aligned}
& \sigma_{a v}=\frac{f(x)}{2 b L}=\frac{f(x)}{\pi R L \cdot \varepsilon_{a v}}, \\
& \sigma_{a v}\left(\varepsilon_{a v}\right)= \begin{cases}\frac{k_{a v} \varepsilon_{a v},}{D_{1} D_{2} \cdot 2 R} & 0 \leq \varepsilon_{a v} \leq \varepsilon_{\lim i t}, \\
\left(D_{2} e^{-\frac{k}{D_{1}} \frac{2 R \varepsilon_{a v}}{v}}+D_{1}\right) \cdot \pi R L & \varepsilon_{\lim i t}<\varepsilon_{a v},\end{cases}
\end{aligned}
$$

where $k_{a v}$ is the stress-strain slope in the linear section, and $\varepsilon_{\lim i t}$ the critical strain at the turning point between the linear section and the Configuration 3 equivalent model.

Using the obtained stress-strain data shown in Figure $7 \mathrm{e}$, simulation experiments were performed combined with Hyperworks and LS-Dyna. The material parameters of the jellyroll are shown in Table 7, and the simulation results are shown in Figure 8.

As shown in Figure 8a-d, the constitutive parameters of the jellyroll obtained by the modified equivalent mechanical model can predict the battery properties well, the simulation effect is better than that of the cubic fitting equation, and the simulation effect of the correlation coefficient $R^{2}$ is larger than 0.9940 .

In addition to the flat plate tests, the rigid rod and hemispherical punch tests are shown in Figure 8e and f. The equivalent mechanical model can reflect the actual situation under different loading conditions, the correlation coefficient $R^{2}$ is larger than 0.9980 , and for the mechanical failure of the battery, the simulation failure displacement can predict the actual failure displacement well. Failure analysis will be introduced in the following section.

In Figure $8 \mathrm{~b}-\mathrm{d}$, the simulation results of medium and high SOCs indicate certain errors in the linear part compared with the experiment. This is primarily because the

Table 7 Material parameter of jellyroll

\begin{tabular}{lllll}
\hline Component & $\begin{array}{l}\text { Young's } \\
\text { modulus } \\
(\mathrm{GPa})\end{array}$ & $\begin{array}{l}\text { Poisson's } \\
\text { ratio }\end{array}$ & $\begin{array}{l}\text { Density }(\mathbf{g} / \\
\left.\mathbf{c m}^{\mathbf{3}}\right)\end{array}$ & TSC (MPa) \\
\hline Jellyroll & 1.5 & 0.15 & 3.22 & 10 \\
\hline
\end{tabular}

Young's modulus of the battery is no longer maintained as a constant value, while the value of $1.5 \mathrm{GPa}$ under $\mathrm{SOC}=0$ is directly used to simplify the simulation process. Hence, the effect of SOC on material constants should be considered in the future to obtain more accurate mechanical properties of the battery.

The simulation results show that the equivalent mechanical model can obtain accurate constitutive parameters, which is beneficial to the development of the simulation and the analysis of battery failure.

\subsection{Failure Analysis under Mechanical Abuse Condition}

During the experiment, the voltage and temperature were measured synchronously and the failure process was recorded. The failure results of the experiment and simulation are shown in Figure 9.

As shown in Figure 9a and b, as the load increased, breaks occurred inside the battery. Under the rigid rod test, the break occurred at the core and bottom of the battery. Under the hemispherical punch test, the break occurred at the core and top of the battery. Figure 9a1 to b2 verify the rationality of the simulation. As shown in Figure 9a1, when the thermal runaway was triggered, the high-temperature point $\mathrm{P0}$ was located at the bottom of the battery. However, the high-temperature point was not observed in the middle of the battery, which exhibited low thermal conductivity in the radial direction. At this time, we removed the punch and the thermal runaway was spread out; after $159.5 \mathrm{~s}$, the highest temperature of $34.94{ }^{\circ} \mathrm{C}$ was reached. Under the hemispherical punch test, similar conclusions were obtained; the hightemperature point P0 was located at the top of the battery, consistent with the simulations. After $143.2 \mathrm{~s}$, the battery reached the highest temperature of $147.7^{\circ} \mathrm{C}$.

As shown in Figure 9e and f, under the rigid rod test at $\mathrm{SOC}=0.6$, when the displacement was approximately $4 \mathrm{~mm}$, the main role of the external load was to squeeze the gap between the battery components such the growth of force was almost linear. During this period, the component structures of the jellyroll-like separator, anode, and cathode have not been destroyed; therefore, the voltage maintained at $3.8 \mathrm{~V}$. Subsequently, the shape of the mechanical experiment curve changed significantly. The curve no longer grew linearly but increased nonlinearly.

When reaching $7.3 \mathrm{~mm}$, the voltage dropped slighted to $3.6 \mathrm{~V}$ at point $\mathrm{A}$, which we call a soft short-circuit. Meanwhile, as shown in the black curve, during the mechanical experiment, mechanical failure point $B$ occurred at the same displacement as point A. The external force resulted in the damage of the battery structure and changed the electrical characteristics of the battery. This proved the possibility of predicting the failure of the battery by mechanical characteristics. 
$\mathbf{a}$

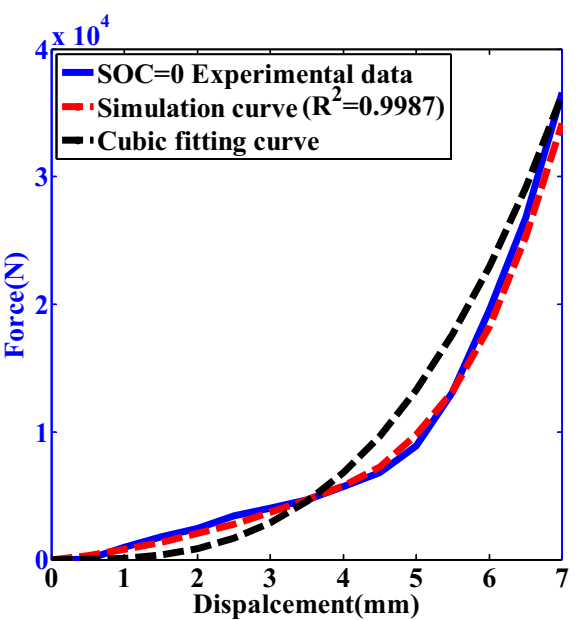

c

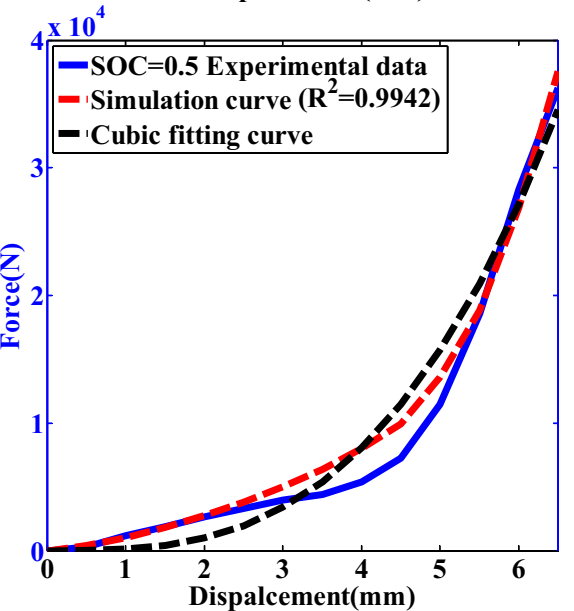

e

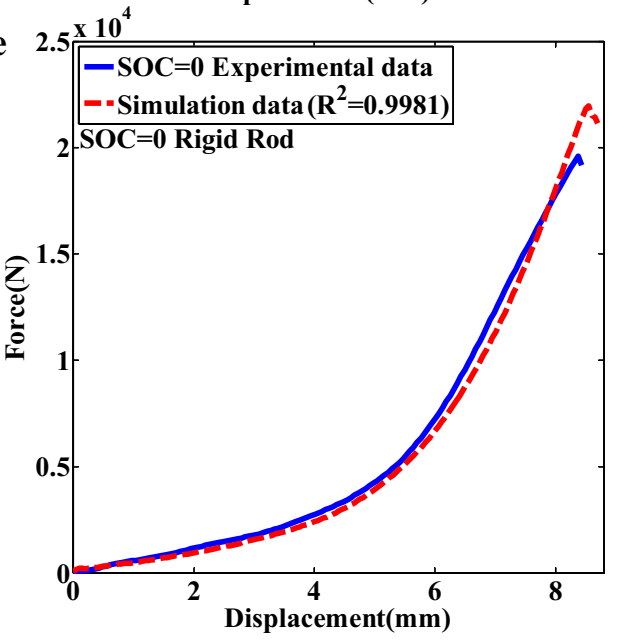

b
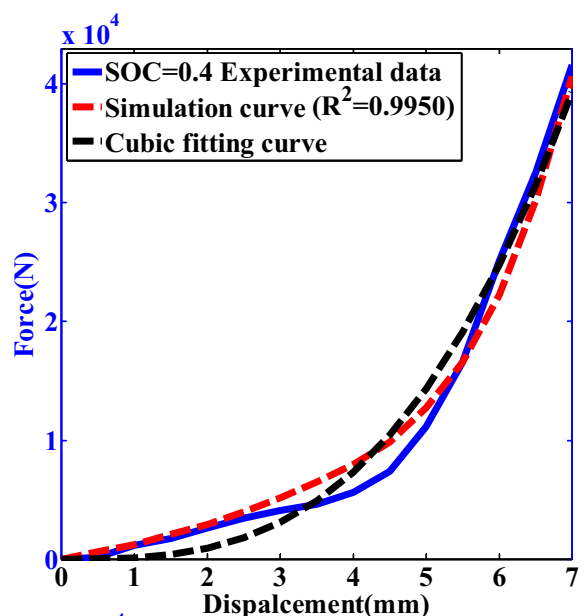

d

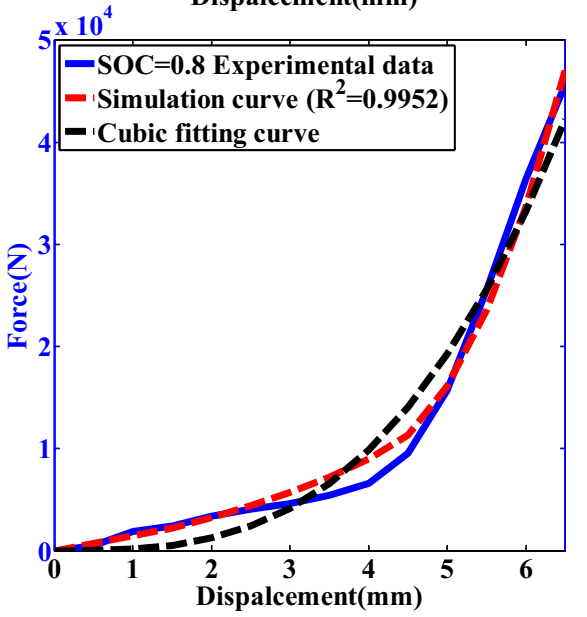

f

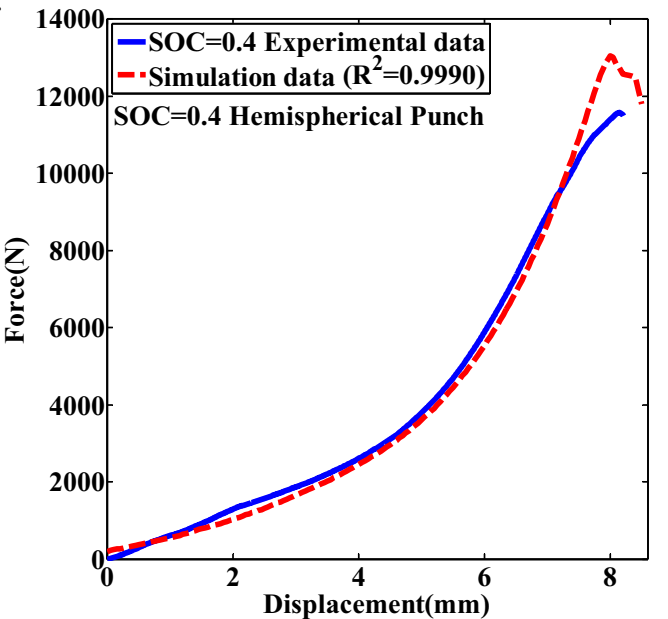

Figure 8 Comparison of experimental and simulation results: $\mathbf{a}-\mathbf{d}$ Flat plates test under $\mathrm{SOC}=0,0.4,0.5$, and 0.8 , e Rigid rod test under $\mathrm{SOC}=0$, $\mathbf{f}$ Hemispherical punch test under $\mathrm{SOC}=0.4$

The soft short-circuit required $19.6 \mathrm{~s}$ to reach $3.6 \mathrm{~V}$; however, the voltage decreased to $0 \mathrm{~V}$ only in $1.65 \mathrm{~s}$ with further compression. By now, the battery has been destroyed completely, and a hard-short circuit occurred.
As shown in Figure 9f, the failure displacement of the battery characterized by electrical results is $7.5 \mathrm{~mm}$, while the simulation failure displacement is $7.65 \mathrm{~mm}$, and the error is only $0.15 \mathrm{~mm}$. This shows that the equivalent 


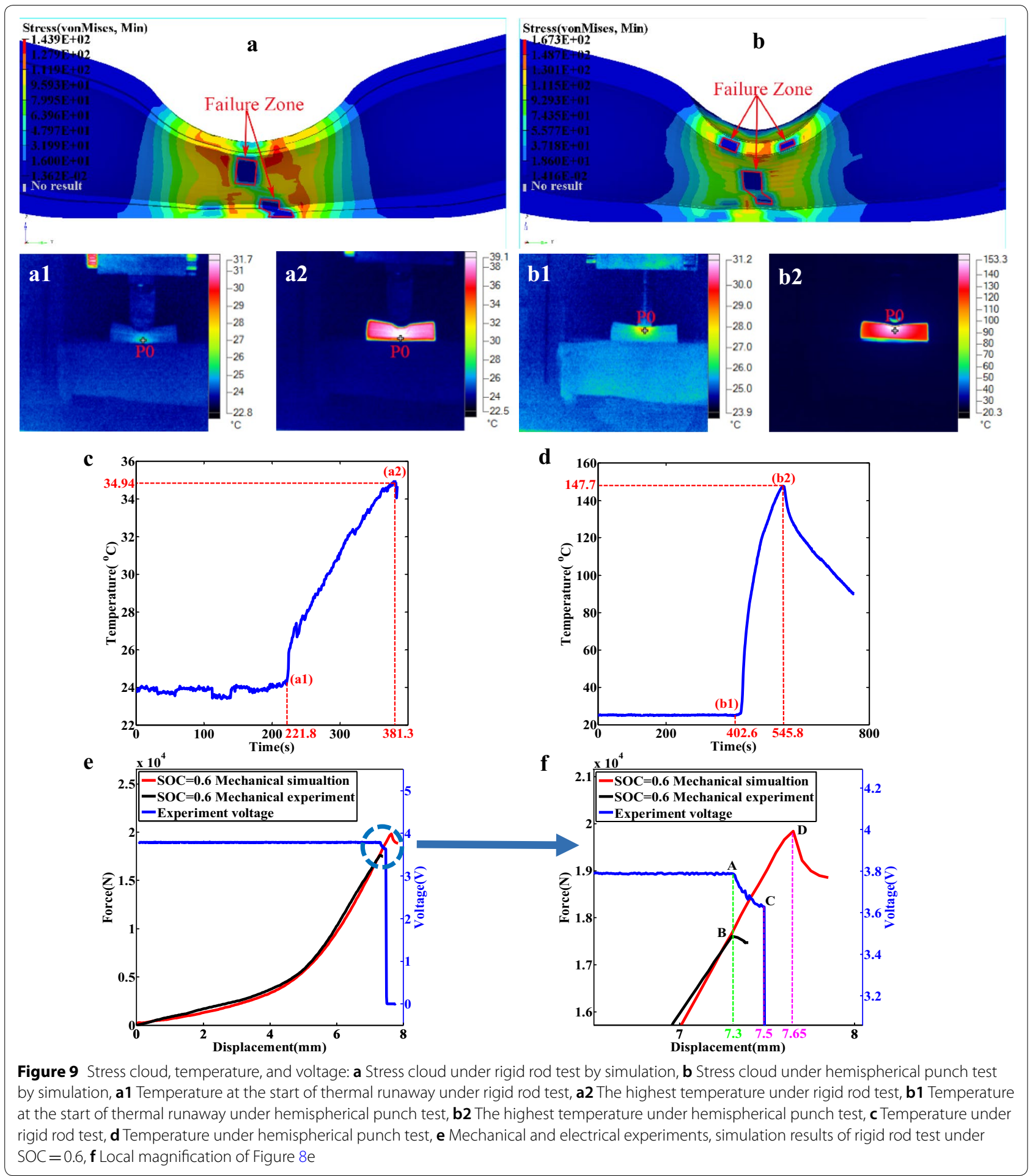

mechanical model can predict the failure displacement of the battery well at the simulation level and proves the practicability of the equivalent mechanical model.

In Table 8, the failure displacement by simulation and experiment under different operating conditions are summarized, from which we discovered that the equivalent mechanical model combined with the crushablefoam material model can effectively predict the failure of the battery under mechanical abuse conditions, and the prediction error is less than $11 \%$. 
Table 8 Failure displacement by experiment and simulation and corresponding relative error

\begin{tabular}{|c|c|c|c|c|c|}
\hline & SOC & 0 & 0.2 & 0.4 & 0.6 \\
\hline \multirow[t]{4}{*}{ Rigid rod test } & Experimental failure displacement (mm) & 8.368 & 8.732 & 8.238 & 7.3 \\
\hline & Simulation failure displacement (mm) & 8.7 & 8.8 & 8.4 & 7.65 \\
\hline & Relative error (\%) & 3.97 & 0.78 & 1.97 & 4.79 \\
\hline & Standard error & 0.33 & 0.06 & 0.36 & 0.35 \\
\hline \multirow[t]{4}{*}{ Hemispherical punch test } & Experimental failure displacement (mm) & 8.9 & 8.35 & 8.15 & 8.45 \\
\hline & Simulation failure displacement (mm) & 8.35 & 8.3 & 8 & 7.6 \\
\hline & Relative error (\%) & 6.18 & 0.60 & 1.84 & 10.06 \\
\hline & Standard error & 0.55 & 0.05 & 0.15 & 0.80 \\
\hline
\end{tabular}

From the experiment, we discovered that the displacement of the change point of the mechanical properties is almost always preceded by the displacement of the change point of the electrical and thermal properties. This suggests that the failure warning by voltage and temperature may lag because the battery energy cannot change immediately when the high capacity battery is subjected to mechanical abuse. Therefore, the detection of battery penetration displacement can be a failure warning mechanism.

\section{Conclusion}

Data analysis indicated that the magnitude-phase-angle characteristics of a battery under mechanical abuse in the frequency domain were similar to those of a springdamping parallel unit. Hence, we proposed an equivalent mechanical model where a spring-damping parallel unit was connected to a damping unit in series with equivalent physical meaning. The modified equivalent mechanical model could reflect the mechanism of the battery under mechanical abuse, the failure occurring at the core and bottom of the battery under a rigid rod test, and the failure occurring at the core and top of the battery under a hemispherical punch test, which were consistent with the experiment.

Through experiments and simulations, we discovered that a certain lag may have occurred in judging the failure of the batteries from in terms of voltage and temperature, i.e., the structure was not heavily damaged, while the internal electrochemical reaction accelerated, and a soft short-circuit occurred. This study suggested that for high-capacity batteries, a lag might have occurred in predicting battery failure merely from electric and temperature. Therefore, future research on failure prediction should focus on the mechanical characteristics.

In summary, the modified equivalent mechanical model provided more accurate constitutive parameters in a simple manner, recognized parameters easily, and presented a reference method for battery modeling and failure analysis. In the future, the model can be used in safety warning devices based on mechanical penetration.

\section{Acknowledgements}

Not applicable.

\section{Authors' Contributions}

WW and $C L$ were in charge of the whole trial; $Y L$ wrote the manuscript and did laboratory analysis; SY assisted with sampling. All authors read and approved the final manuscript.

\section{Authors' Information}

Wenwei Wang, born in 1980, received his PhD degree from School of Mechanical Engineering, Beijing Institute of Technology, China, in 2007. He is an Associate Professor at School of Mechanical Engineering, the Assistant Dean for New Energy Automotive Research Institute of Beijing Institute of Technology, the Deputy Director of Beijing Engineering Research Center of Electric Vehicle

Yiding Li, born in 1993, received his B.E. degree in Mechanical engineering and automation from Shandong University, China, 2016. He is a PhD candidate in mechanical engineering at National Engineering Laboratory for Electric Vehicles, Beijing Institute of Technology, China. His main research interest is in the area of the battery safety.

Cheng Lin, born in 1968, received his B.S. and M.S. degree in Mechanical Department from Wuhan Institute of Technology, China, in 1990 and 1995, respectively. He graduated from School of Mechanical Engineering, Beijing Institute of Technology, China, in 2002 with PhD degree. He is a Professor/PHD Tutor at School of Mechanical Engineering, Beijing Institute of Technology, China, the Deputy Director of National Engineering Laboratory for Electric Vehicle, the Director of Research Center for Electric Vehicle in Beijing.

Sheng Yang, born in 1991, is currently a PhD candidate at National Engineering Laboratory for Electric Vehicles. Beijing Institute of Technology, China.

\section{Funding}

Supported by National Key R\&D Program of China (Grant No. 2017YFB0103801)

\section{Competing Interests}

The authors declare no competing financial interests.

\section{Author Details}

${ }^{1}$ National Engineering Laboratory for Electric Vehicles, Beijing Institute of Technology, Beijing 100081, China. ${ }^{2}$ Collaborative Innovation Center of Electric Vehicles in Beijing, Beijing Institute of Technology, Beijing 100081, China.

Received: 11 July 2019 Revised: 7 January 2020 Accepted: 14 February 2020

Published online: 02 March 2020 


\section{References}

[1] P Peng, F M Jiang. Thermal safety of lithium-ion batteries with various cathode materials: A numerical study. J. Heat Mass Tran., 2016, 103: 1008-1016

[2] J G Kim, B Son, S Mukherjee, et al. A review of lithium and non-lithium based solid state batteries. J. Power Sources, 2015, 282: 299-322.

[3] XW Zhang, E Sahraei, KWang. Li-ion battery separators, mechanical integrity and failure mechanisms leading to soft and hard internal shorts. Sci. Rep., 2016, 6, 32578, https://doi.org/10.1038/srep32578.

[4] A Sheidaei, X R Xiao, X S Huang, et al. Mechanical behavior of a battery separator in electrolyte solutions. J. Power Sources, 2011, 196: 8728-8734.

[5] E P Roth, D H Doughty, D L Pile. Effects of separator breakdown on abuse response of 18650 Li-ion cells. J. Power Sources, 2007, 174: 579-583.

[6] TWierzbicki, E Sahraei. Homogenized mechanical properties for the jellyroll of cylindrical Lithium-ion cells. J. Power Sources, 2013, 241: 467-476.

[7] I Avdeev, M Gilaki. Structural analysis and experimental characterization of cylindrical lithium-ion battery cells subject to lateral impact. J. Power Sources, 2014, 271: 382-391.

[8] W W Wang, S Yang, C Lin. Clay-like mechanical properties for the jellyroll of cylindrical Lithium-ion cells. Applied Energy, 2017, 196: 249-258.

[9] E Sahraei, J Meier, TWierzbicki. Characterizing and modeling mechanical properties and onset of short circuit for three types of lithium-ion pouch cells. J. Power Sources, 2014, 247: 503-516.

[10] C Zhang, J Xu, L Cao, et al. Constitutive behavior and progressive mechanical failure of electrodes in lithium-ion batteries. J. Power Sources, 2017, 357: 126-137.

[11] S H Chung, TT Dejean, et al. Failure in lithium-ion batteries under transverse indentation loading. J. Power Sources, 2018, 389: 148-159.

[12] J Xu, B H Liu, L B Wang, et al. Dynamic mechanical integrity of cylindrical lithium-ion battery cell upon crushing. Engineering Failure Analysis, 2015, 53: 97-110.

[13] E Sahraei, R Hill, TWierzbicki. Calibration and finite element simulation of pouch lithium-ion batteries for mechanical integrity. J. Power Sources, 2012, 201: 307-321.

[14] C Zhang, S Santhanagopalan, M A Sprague, et al. Coupled mechanicalelectrical-thermal modeling for short-circuit prediction in a lithium-ion cell under mechanical abuse. J. Power Sources, 2015, 290: 102-113.

[15] L Greve, C Fehrenbach. Mechanical testing and macro-mechanical finite element simulation of the deformation, fracture, and short circuit initiation of cylindrical Lithium ion battery cells. J. Power Sources, 2012, 214 377-385.

[16] E Sahraei, J Campbell, TWierzbicki. Modeling and short circuit detection of 18650 Li-ion cells under mechanical abuse conditions. J. Power Sources, 2012, 220: 360-372
[17] J E Zhu, XW Zhang, E Sahraei, et al. Deformation and failure mechanisms of 18650 battery cells under axial compression. J. Power Sources, 2016, 336: 332-340.

[18] S Kim, Y S Lee, H L Jin. A study on the behavior of a cylindrical type Li-lon secondary battery under abnormal conditions. Mat. - wiss. u. Werkstofftech, 2010, 41(5): 378-385.

[19] E Sahraei, E Bosco, B Dixon, et al. Microscale failure mechanisms leading to internal short circuit in Li-ion batteries under complex loading scenarios. J. Power Sources, 2016, 319: 56-65.

[20] B H Liu, Y K Jia, J Li, et al. Safety issues caused by internal short circuits in lithium-ion batteries. Journal of Material Chemistry A, 2018, 6: 21475.

[21] J E Zhu, TWierzbicki, W Li. A review of safety-focused mechanical modeling of commercial lithium-ion batteries. J. Power Sources, 2018, 378: 153-168.

[22] J Xu, B H Liu, XY Wang, et al. Computational model of 18650 lithium-ion battery with coupled strain rate and SOC dependencies. Applied Energy, 2016, 172: 180-189.

[23] J Xu, B H Liu, D Y Hu. State of charge dependent mechanical integrity behavior of 18650 lithium-ion batteries. Sci. Rep., 2016, 6, 21829, https:// doi.org/10.1038/srep21829.

[24] H L Luo, Y Xia, Q Zhou. Mechanical damage in a lithium-ion pouch cell under indentation loads. J. Power Sources, 2019, 357: 951-960.

[25] Y K Jia, S Yin, B H Liu, et al. Unlocking the coupling mechanical-electrochemical behavior of lithium-ion battery upon dynamic mechanical loading. Energy, 2016, 166: 278-289.

[26] M Ichimura. The safety characteristics of lithium-ion batteries for mobile phones and the nail penetration test. 29th International Telecommunications Energy Conference, 2007: 687-692.

[27] B H Liu, H Zhao, H L Yu, et al. Multiphysics computational framework for cylindrical lithium-ion batteries under mechanical abusive loading. J. Power Sources, 2017, 256: 172-184.

[28] C Zhang, S Santhanagopalan, M A Sprague, et al. A representative-sandwich model for simultaneously coupled mechanical-electrical-thermal simulation of a lithium-ion cell under quasi-static indentation tests. J. Power Sources, 2015, 298: 309-321.

[29] V S Deshpande, N A Fleck. Isotropic constitutive models for metallic foams. J. the Mechanics and Physics of Solids, 2000, 48: 1253-1283.

[30] W W Wang, Y D Li, C Lin, et al. State of charge-dependent failure prediction model for cylindrical lithium-ion batteries under mechanical abuse. Applied Energy, 2019, 251: 113365

\section{Submit your manuscript to a SpringerOpen ${ }^{\circ}$ journal and benefit from:}

- Convenient online submission

- Rigorous peer review

- Open access: articles freely available online

- High visibility within the field

Retaining the copyright to your article

Submit your next manuscript at springeropen.com 